\title{
Online Pragmatic Language Use in Asperger Syndrome and Learning Disability Discussion Forums
}

\author{
Francesca Dansereau ${ }^{1}$ \\ Tara Flanagan ${ }^{2}$ \\ Department of Educational and Counselling Psychology, McGill University, Canada \\ :Email:francesca.dansereau@mail.mcgill.ca
}

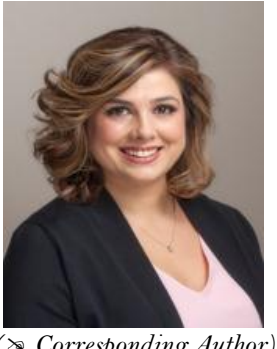

\section{Abstract}

Research has indicated that self-expression for individuals on the autism spectrum may be facilitated through written language by promoting a more flexible environment within which to express beliefs, opinions, ideologies and expectations-in essence, creating a safe place for communication. Such safe places have burgeoned with the evolution of the Internet, and many individuals with autism have taken the opportunity to express themselves publicly by developing their own virtual communities, some of which have taken place on discussion forums, websites and weblogs. This research study evaluates natural discourse in an Asperger Syndrome discussion forum to explore social communication in the autism spectrum community by using the pragmatic profile questionnaire of the CELF. A discussion forum for individuals with learning disabilities was used to explore similarities and differences in online pragmatic language use among people who self-identified as belonging to one of the two groups. The findings highlight the nuances in pragmatic language use in online discussion forums and the need to consider these skills in context. Researchers and practitioners should refrain from making one-size-fits-all recommendations regarding the creation of supportive social spaces for individuals on the autism spectrum.

Keywords: Asperger syndrome, Learning disabilities, Pragmatic language, Online discussion forums.

JEL Classification: Ioo Health, Education and Welfare: General.

Citation | Francesca Dansereau; Tara Flanagan (2019). Online Pragmatic Language Use in Asperger Syndrome and Learning Disability Discussion Forums. Asian Journal of Social Sciences and Management Studies, 6(1): 1-6.

\section{History:}

Received: 18 October 2018

Revised: 15 November 2018

Accepted: 31 December 2018

Published: 21 January 2019

Licensed: This work is licensed under a Creative Commons

Attribution 3.0 License (cc) Er

Publisher: Asian Online Journal Publishing Group
Contribution/Acknowledgement: Both authors contributed to the conception and design of the study.

Funding: This study received no specific financial support.

Competing Interests: The authors declare that they have no conflict of interests.

Transparency: The authors confirm that the manuscript is an honest, Transparency: The authors confirm that the manuscript is an honest,
accurate, and transparent account of the study was reported; that no vital accurate, and transparent account of the study was reported; that no vital
features of the study have been omitted; and that any discrepancies from the study as planned have been explained.

Ethical: This study follows all ethical practices during writing.

\section{Contents}

1. Introduction

2. Methodology

3. Results.

4. Discussion.

5. Limitations

6. Conclusions and Future Directions

References.. 


\section{Introduction}

The oral and written modalities differ in many different ways. The main difference concerns the processing speed of the speaker and the listener. In spoken language, the processing is initiated from the time the speaker states their first word and stops when they finish their statement. It is only at this time that the listener validates their understanding or interpretation of what was stated, which demands a certain amount of short-term memory usage. According to Alloway et al. (2009) children with Asperger syndrome show difficulties with verbal shortterm memory, a component of oral language. Asperger syndrome, considered under the umbrella of Autism spectrum disorder (ASD), is a neurological disorder that impacts social interaction and communication across multiple contexts (American Psychiatric Association, 1994).

Short-term memory for verbal material is essential in reciprocal speech; one must remember what is said in order to respond appropriately. However, the written modality permits the writer to process the information on their timeline as they may reread the information as many times as necessary, until the recipient receives the message. This process increases the possibility of comprehension and reduces the processing speed of the reader when compared to the listener in the oral modality (Auer, 2009). This processing delay is considered a main reason why individuals on the autism spectrum are able to increase their communication capabilities within the written modality (Schairer and Nelson, 1996; Alloway et al., 2009).

In addition to the research findings that support this notion, anecdotal evidence, such as autobiographies, provide further explanation as to why the written modality is favoured by individuals on the autism spectrum. Two individuals on the autism spectrum write; "I still have problems in groups where there is too much information going on, or where the informational flow is too fast. I simply can't shift my attention quickly enough to the relevant part of the interaction process. Consequently, I miss a lot of vital information needed to interpret social messages. There is no such thing as adequate delayed social reactions. One either is quick enough to keep up, or one is weird and socially disabled" (Prince-Hughes, 2002). "I find the written word much easier to comprehend than the spoken word. It takes me a lot longer to process conversation and work out the meaning behind the words than it does to scan the words on a written page. I think this is because I must read the expressions on a person's face and study their body language" (Lawson, 1998).

\subsection{The Written Modality Facilitates Communication}

The written modality, facilitating communication for those on the autism spectrum, has increased their possibilities for self-expression and advocacy. In their research, Schairer and Nelson (1996) found that the written modality increases the communication capabilities for individuals who are on the autism spectrum since the sentence length was significantly higher in the written modality even though the number of utterances was greater in the oral modality; meaning that spoken language occurs more rapidly than written language. For example, their sentence structure in the written modality was more complex than their short to the point spoken answers in the oral modality. They also noticed that pragmatics such as "turn-taking" and "topic matching," showed no significant difference. Concluding that the written modality provided a better communication medium even if the number of utterances were exceeded in the oral modality (Schairer and Nelson, 1996).

The research article by Schairer and Nelson (1996) is the only study providing empirical quantitative evidence between the oral and written modality among individuals on the autism spectrum. In a study exploring the written modality, the Linguistic Inquiry and Word Count dictionaries were used to compare the written language of individuals on the autism spectrum to neurotypical individuals (Newton et al., 2009). No differences were found in the rate of word usage between both groups with the small exception that there was more variation in social language use individuals with ASD (Newton et al., 2009). Evidence from personal accounts seems to corroborate these findings of facility with the written word among individuals on the autism spectrum. "I have no problems expressing my emotions on paper at all" (Prince-Hughes, 2002). "I could write essays all day - they do not bite back at me. The written word has a form all its own. The pen between my fingers feels solid and tangible. It moves with me and allows the symbols of my pain or ecstasy to reveal themselves. Words express my distress through the pen and onto the paper and back into my mind. I can see them on the paper; they talk to me and help me to make sense of my life. .. . Then words are pure and not distorted by grimaces and gestures. I can listen better to the tone of someone's voice when I am not confused by the unwritten words of their facial expressions" (Lawson, 1998). "On paper, [...words poured out of me. I liked writing and was very good at spelling - in fact I simply couldn't make spelling mistakes" (Gerland, 1996).

These autobiographies provide evidence that the written modality has facilitated abilities related to selfexpression by providing an environment that is better built for communication. Further, written expression may help provide an opportunity to gain some control over one's often over-stimulating environment. According to Davidson (2007) awkward behaviour within the neurotypical environment might be caused by a discomfort that becomes expressed as a self-defense mechanism (Davidson, 2007). For instance, one individual on the spectrum writes, "this oversensitive hearing is often a problem because sounds that other people don't even notice are disturbing and even painful to me. I have huge problems filtering out what I want to hear. For comprehension, I tend to rely on context a lot. This type of hearing problem makes social life very tiring, because as soon as there are more than two people present, listening to conversations means having to put in a lot of effort piecing together what people might be saying" (Prince-Hughes, 2002). Every person on the autism spectrum deals with these overstimulating environments differently (Rutter et al., 2005) though computer-mediated communication (Jones et al., 2001; Newton et al., 2009) and written expression might serve as vehicles of effective coping and communication, as well as community-building. .

\subsection{Online Communities and Discussion Forums}

Individuals on the autism spectrum use the written modality across a variety of online platforms to express their feelings, to have important discussions and debates about "autism," and to combat stigmatization (Chamak, 2008). These discussion forums seem to provide a way for individuals on the autism spectrum to socialize with other individuals who have autism or Asperger syndrome, to create safe spaces, and to actively participate in their 
communities. For instance, an increasingly popular online association for individuals who have autism called "Autism Network International" has not obtained a significant amount of media coverage, but is continuously expanding through the Internet. It is renown as the first community for individuals on the autism spectrum and continues to be the largest organization of its kind (Sinclair, 2005). According to Chamak (2008) the Internet has facilitated the exchange of ideas and opinions that can otherwise be difficult to establish for individuals on the autism spectrum and has facilitated the development of online and offline social networks (Sinclair, 2005).

\subsection{This Study}

In line with the views of the oral versus the written modality mentioned earlier in this paper, this study explores natural discourse of individuals on the autism spectrum in an asynchronous discussion forum. In accordance with the research conducted by Brownlow and O'Dell (2006) and Newton et al. (2009) the written modality should facilitate social communication for a number of reasons; removal of face-to-face didactic conversation (Blume, 1997) removal of an over stimulating environment (Davidson, 2007); (Rutter et al., 2005) and the ability to re-read their response providing them with a processing delay that would be required in social auditory language (Schairer and Nelson, 1996; Prince-Hughes, 2002; Auer, 2009).

The main purpose of this research study is to contribute to the empirical data related in exploring the online social communication of individuals on the autism spectrum by using a standardized measure of pragmatic language and by choosing a suitable comparison group. Pragmatic language is an umbrella term for the language abilities that are specifically tied to communication (e.g., initiating conversations, understanding inferences and sarcasm, staying on topic and asking appropriate questions, and engaging in reciprocal conversations) (Semel et al., 2003). The comparison group for this study is comprised of individuals who identify as having a learning disability (LD) (with and without attention deficit (ADD) or attention deficit hyperactivity disorder (ADHD))). Similarly to individuals with ASD those with an LD also follows a spectrum (Mayes et al., 2000) and may equally display difficulties with self-expression. The participants who disclosed a possible diagnosis of ADD or ADHD, even without the comorbidity of an LD, were not been excluded from this study since they also express difficulties with processing speeds (Chhabildas et al., 2001). In addition, the same way that autism spectrum disorder is diagnosed 4 times more frequently in males than in females, ADD and ADHD have both been shown to be more prevalent among males than females (APA, 2013). Therefore, it was believed that the LD group was a good comparison match. As the diversity in the ASD community is impossible to capture in one study, only individuals who selfidentified as Asperger (Aspies) were included.

Previous research studies stipulate that individuals on the autism spectrum benefit from online platforms for self-expression and self-advocacy purposes (Gerland, 1996; Schairer and Nelson, 1996; Lawson, 1998; PrinceHughes, 2002; Semel et al., 2003; Brownlow and O'Dell, 2006; Chamak, 2008). Therefore, this paper explores the online pragmatic language use of individuals on the autism spectrum by comparing their natural written discourse and comparing it to individuals with an LD and/ or ADD/ ADHD. This will be accomplished by using the "pragmatic language profile questionnaire" of the Clinical Evaluation of Language Fundamentals-Fourth Edition (CELF) to better determine which aspects of social language are implicated in online social communication in discussion forums among the two groups. This study is exploratory in nature and will hopefully contribute empirical data to an emerging field.

\section{Methodology}

Participants. 19 individuals who self-identified as having a diagnosis of Asperger Syndrome (AS) and 9individuals who self-identifies as having a diagnosis of a learning and/or an attention disability (LD) participated in this study via publicly available discussion forums. Due to online anonymity, the individuals' complete demographics cannot be reported for this study. In addition, all participants who posted on multiple occasions, meaning more than once across the posts selected, were removed from the study. Furthermore, one individual in the LD group and two from the AS group were removed from the study since inter-rater reliability was not established for the content of their discussions.

Instruments. To create a pragmatic profile for each participant, the observation questionnaire called "Pragmatics Profile" of the Clinical Evaluation of Language Fundamentals-Fourth Edition (CELF) was administered. The clinical evaluation of language fundamentals fourth edition (CELF-4) is a psychometric assessment that was published in 1980 and was designed to identify, diagnose, and evaluate language and communication disorders in learners between 5 to 21 years of age. It facilitates an adaptable, multi-perspective assessment process that distinguishes a learner's language and communication strengths and weaknesses, and provides educationally pertinent interventions and accommodations (Semel et al., 2003).

Procedure. For the Asperger group, the asynchronous discussion forum used was called "Aspies for Freedom." As a passive observer, the main researcher randomly chose five different conversations that seemed to have the most replies by the participants. The topics of conversations were not considered, since we were solely assessing the nature of the social conversation and not the specific topics listed. To determine the actual difference in social interaction, the asynchronous discussion forum of the comparison group was called, "Learning Disability Support Group". Both of these discussion forums had to meet the following criteria in order to be used in this study: 1) be asynchronous discussion forums, 2) the person with the disability participated in discussions and not their parent(s) or friend(s), 3) be accessible via major search engines (i.e.: google.com). Five conversations having the highest number of posts were reviewed at random from both forums.

Both discussion forums were not closed to the public, but fully accessible through google.com search engine. The active participants had to be members of the forums in order to post, but the general public has full access to read any post or overall discussion. The researchers remained observers throughout the duration of the study and never registered or posted as a way to add to conversation. The discussions selected had to meet the following criteria: 1) identifying with a diagnostic group that is considered to be a spectrum, 2) the individual with a diagnosis is the one participating in conversation, 3) the individual must mention in one of their posts that they are either on the autism spectrum or have an LD (any type), 4) the individuals are expressing their opinions or beliefs 
or emotions, and 5) the conversations had to be longer than two postings. Each observer rated each participant using the "Pragmatic Profile Questionnaire" of the CELF.

To explore naturally developing online discourse, this study used already existing discussions instead of using a pilot with consented forums. Since there are no guidelines for using this information for research purposes, the participants of this study were not contacted prior to the analysis of their posts (Brownlow and O'Dell, 2002). Many researchers have discussed the ethical issues related to collecting qualitative data through open sources such as blogs, discussion forums, and other social networking sites (Brownlow and O'Dell, 2002; Berry, 2004) but they do not discuss the issues concerning quantitative data collection as the main concern is regarding how to maintain the confidentiality and safety of the participant (Brownlow and O'Dell, 2002).

The subtest, the Pragmatic Profile observation questionnaire, of the CELF was the only psychometric assessment used in this study since it provides an overview of the pragmatics in social communication situations. Observing only the written aspects of language, all questions relating to body movements such as eye contact, appropriate body posture during conversation, and the likes have been rated as "not applicable”. To properly establish inter-rater reliability, prior to analysis of the posts, a rating criteria list was established among the three evaluators (the main researcher and two raters who were blind to the diagnoses and purpose of the study).

\section{Results}

To ensure reliability among the three ratters when using the CELF, a Kappa calculator was used since it calculates the chance-adjusted measure of agreement between any number of cases, categories and ratters. The calculator provided two types of variations, Siegel and Castellan (1988) fixed-marginal multi-rater as well as Randolph's free-marginal multi-rater (Randolph, 2005; Warrens, 2010). The Kappa values can range between -1.0 to 1.0, where -1.0 indicates perfect disagreement below chance, 0.0 agreements equal to chance, and 1.0 perfect agreement above chance. Adequate inter-rater agreement must be 0.7 or above.

After including only the discussions where inter-rater reliability was established according to the Kappa calculator, an ANOVA was conducted to see if there was a significant difference between the Kappa's found between groups (AS vs. LD). After this analysis no significant difference was found suggesting high inter-rater reliability $(p=.162, p<0.5)$.

The data collected were on a likert scale (O-4) so there was little variance among scores in such that there were no outliers. In addition, there were no missing data as each rater completed each of the questions on each pragmatic profile. Furthermore, the responses of each profile predictor were skewed. There were more positively skewed predictors (observes turn-taking rules in the classroom or in social interactions $(z=1.05)$, introduces appropriate topics of conversation $(z=.34)$, asks appropriate questions during conversations and discussions $(z=1.49)$, asks for/ responds for clarification during conversation $(z=5.14)$, uses the language (jargon/ lingo) of his/her peer group appropriately $(z=1.05)$, uses appropriate strategies for getting attention $(z=5.43)$, asks for help from others appropriately $(z=7.62)$, offers to help others appropriately $(z=7.99)$, gives/ responds to advice or suggestions appropriately $(z=1.90)$, asks for clarification if he/ she is confused or if the situation is unclear $(z=$ 7.99), offers/ responds to expressions of affection $(z=5.43)$ ) than negatively skewed predictors (begins/ ends conversations appropriately $(z=-12.01)$, maintains topic using appropriate strategies $(z=-12.01)$, makes relevant contributions to a topic during conversation and discussion $(z=-12.01)$, adjusts/ modifies language based on the communication situation $(z=-12.01)$, tells/ understands jokes/stories that are appropriate to the situation $(z=-$ $3.34)$, joins or leaves an ongoing communicative interaction appropriately $(z=-7.99)$, gives/ asks for reasons and causes for actions/ conditions/ choices $(z=-4.75)$ ). A log, square root, and inverse transformation were attempted as a way to deal with the skewness, but all three methods were ineffective. Each of these predictors demonstrated significant differences amongst scores especially when considering the frequencies of responses. This should not be surprising as the two groups are different and this should affect their communication profiles.

Between Group Comparisons. A frequency count was used to explore the instances of behaviours related to each question of the Pragmatic Profile questionnaire of the CELF (Please see Table 1). As some participants were involved in more than one discussion and in order to better equate the groups, group differences are discussed only for individuals who participated in only one discussion. Both groups demonstrated a large number of skills as assessed by the CELF.

The Asperger group demonstrated higher frequencies related to maintaining conversation, leaving and joining conversation, giving/ asking for reasons and causes for actions/ conditions/ choices, asking for help from others appropriately, and agreeing and disagreeing using appropriate language. However, the LD group demonstrated higher frequencies related to being able to better begin and end conversations, turn-take, make relevant contributions to conversation, and to use appropriate strategies for getting attention. Both groups were able to avoid the use of repetitive language, showed appropriate sense of humour, and asked for clarification if they were confused or if the situation is unclear.

More specifically, the Asperger group showed differences when introducing appropriate topics of conversation since they were not always appropriate in comparison to the LD group who maintained appropriateness throughout. Even though it was more observable throughout the Asperger group than the LD group, it was noticed that the Asperger group asks appropriate questions more frequently during conversations and discussions than the LD group, but the LD group used language (jargon/ lingo) of his/her peer group appropriately more frequently than the Asperger group. Although the LD group showed a percentage of $11.1 \%$ when asking for/ responding to clarification during conversation, the Asperger group ranged between 10.5\% for "often" and 5.3\% for "always"; showing that the Asperger group was able to do this more frequently than the LD group. The LD group was able to adjusts/ modifies language based on the communication situation all the time whereas the Asperger group ranged between $5.3 \%$ for "often" and " $94.7 \%$ " for always. It was noticed that the LD group offers to help others appropriately whereas in the Asperger group this part of pragmatics was never observed. A small difference was noticed between groups when assessing telling/ understanding jokes/ stories (AS = 78.9\% and LD = 77.8\%). The Asperger group ranged between "often" and "always" for giving/ responding to advice or suggestions appropriately, whereas the LD group maintained a percentage in the "always” column. However, the LD group 
ranged between "sometimes" and "always" for offering/ responding to expressions of affection, appreciation appropriately, whereas the Asperger group maintained a percentage in the "always" column. Although some of these are slight discrepancies, it shows that different pragmatic aspects were better reached among one group than another demonstrating slight discrepancies on the overall usage of language between groups.

To further explore pragmatic language profiles a cluster analysis was conducted to determine the similarities or differences within and between the two groups. Similarly to studies assessing pragmatic language profiles by either using the CELF-4 assessment (Lewis et al., 2006) or the Western Aphasia Battery (Lewis et al., 2007) we conducted a hierarchical cluster analysis. Four types of clusters were formed within both groups (LD and ASD). Within the LD group, the four clusters were divided so that 6 participants were part of cluster 1 and the other 3 clusters only contained 1 participant each. This shows that 6 out of the 9 participants had similar online pragmatic language profiles whereas 3 remain distinct from the others. The clusters within the AS groups were slightly more varied when compared to the LD group; cluster 1 contained 13 participants, cluster 2 contained 1 participant, cluster 3 contained 2 participants, and cluster 4 contained 3 participants. This shows the diversity among the AS group. These results highlight that the common clinical practice of creating profiles that can later become associated with particular types of interventions should be used with caution as our online pragmatic language profiles differed between and within groups.

\section{Discussion}

The main purpose of this research was to explore the online pragmatic language of individuals on the autism spectrum in relation to individuals with learning disabilities to contribute empirical data in the area. This was an initial step toward better understanding the potential of online forums to be flexible and facilitative spaces for social communication among individuals on the autism spectrum.

The main finding from this research is that group differences in pragmatic language did still exist even in an environment that has been discussed as facilitative for social communication. The AS and LD groups displayed rather different profiles of pragmatic language even through the use of the written modality. In general the Asperger members were able to introduce appropriate topics of conversation, and avoid using repetitive/redundant information when participating in one or multiple discussions. The LD group members used many more components of pragmatic language within their discussions than the Asperger group, but were still lacking in different areas. For instance, in the one group discussion it was noticed that the Asperger group was better at maintaining conversation, leaving and joining conversation, giving/ asking for reasons and causes for actions/ conditions/ choices, asking for help from others appropriately, and agreeing as well as disagreeing using appropriate language. There is not enough evidence to suggest that the AS group showed more difficulty with pragmatic language, but it seems that the AS and LD may use pragmatic language for different purposes in online discussion forums. Both groups demonstrated a distinct pragmatic language profile with higher frequencies of certain components of the CELF being salient. The hierarchal cluster analysis furthered the notion that diversity exists both between groups (AS and LD), but also within each group. Therefore, there is no distinct pragmatic language profile for each group based on a particular diagnosis, nor would there be a one-size-fits-all approach to addressing difficulties in social communication.

\section{Limitations}

This exploratory study using asynchronous discussion forums as a primary source of data was limited by the necessity of participant anonymity. Though there were instances where demographic information such as age, education, and marital status were mentioned, they were not recorded. Though the pragmatic profile questionnaire of the CELF was deemed the most appropriate measure for the purposes of this study, we were not able to adhere to the recommendation that it should be administered by an examiner who knows the participant well (Semel et al., 2003). Thus, some of the nuances of pragmatic language use by individual participants may have been missed due to the objectivity of the observers. Finally, passive observation in online forums is limited by the inability to validate the information (e.g., diagnosis) provided by the participants.

\section{Conclusions and Future Directions}

At the start, we noted that individuals on the autism spectrum (here the AS group) might benefit from discussion forums and asynchronous social networking sites since these environments remove face-to-face didactic conversation (Blume, 1997) over-stimulating environments (Rutter et al., 2005; Davidson, 2007) and provide the speech delay required to better process information (Schairer and Nelson, 1996; Prince-Hughes, 2002; Auer, 2009). Previous studies have noted increased use of pragmatic vocabulary on social networking sites among individuals on the autism spectrum (Newton et al., 2009) and that online forums facilitate the expression of opinions (Brownlow and O'Dell, 2006) and of feelings and emotions (Jones et al., 2001). However, previous studies have largely neglected to study naturally developing discourse in asynchronous discussion forums, have not included standardized measures of pragmatic language, and have not used comparison groups. In contrast, we explored online pragmatic language use in AS and LD discussion forums using the Pragmatic Profile questionnaire of the CELF, and found quite a bit of between and within group diversity. Future research could build on this work by exploring the barriers and facilitators of online pragmatic language use among individuals on the autism spectrum by making comparisons using different reference groups, and by studying the motivations behind and experiences with participating in online forums. Our findings, though exploratory in nature, highlight the need to recognize the diversity inherent to pragmatic language use, provide initial support for the use of standardized measures in online research, and provide an example of how to incorporate comparison groups in online research. Researchers and practitioners should distance themselves from one-size-fits-all approaches to working with individuals on the autism spectrum and find ways to create safe spaces that capitalize on the diversity in the community. 


\section{References}

Alloway, T.P., G. Rajendran and L.M. Archibald, 2009. Working memory in children with developmental disorders. Journal of Learning Disabilities, 42(4): 372-382.

American Psychiatric Association, 1994. Diagnostic and statistical manual of mental disorders. 5th Edn., Washington, DC: Author.

APA, 2013. Diagnostic and statistical manual of mental disorders. 5th Edn., Washington, DC: Author.

Auer, P., 2009. On-line syntax: Thoughts on the temporality of spoken language. Language Sciences, 31(1): 1-13. Available at: https://doi.org/10.1016/j.langsci.2007.10.004.

Berry, D.M., 2004. Internet research: Privacy, ethics and alienation: An open source approach. Internet Research, 14(4): 323-332. Available at: https://doi.org/10.1108/10662240410555333.

Blume, H., 1997. Autistics are communicating in cyberspace. Available from http://nytimes.com/library/cyber/techcol/063097techcol.html

Brownlow, C. and L. O'Dell, 2002. Ethical issues for qualitative research in on-line communities. Disability \& Society, 17(6): 685-694. Available at: https://doi.org/10.1080/0968759022000010452.

Brownlow, C. and L. O'Dell, 2006. Constructing an autistic identity: AS voices online. Mental Retardation, 44(5): $315-321$.

Chamak, B., 2008. Autism and social movements: French parents' associations and international autistic individuals' organisations. Sociology of Health \& Illness, 30(1): 76-96. Available at: https://doi.org/10.1111/j.1467-9566.2007.01053.x.

Chhabildas, N., B.F. Pennington and E.G. Willcutt, 2001. A comparison of the neuropsychological profiles of the DSM-IV subtypes of ADHD. Journal of Abnormal Child Psychology, 29(6): 529-540.

Davidson, J., 2007. 'In a World of her Own...': Re-presenting alienation and emotion in the lives and writings of women with autism. Gender, Place and Culture, 14(6): 659-677. Available at: https://doi.org/10.1080/09663690701659135.

Gerland, G., 1996. A real person: Life on the outside. London: Souvenir Press.

Jones, R.S., A. Zahl and J.C. Huws, 2001. First-hand accounts of emotional experiences in autism: A qualitative analysis. Disability \& Society, 16(3): 393-401. Available at: https://doi.org/10.1080/09687590120045950.

Lawson, W., 1998. Life behind glass: A personal account of autism spectrum disorder. London \& New York: Jessica Kingsley Publishers.

Lewis, F.M., B.E. Murdoch and G.C. Woodyatt, 2006. Linguistic abilities in children with autism spectrum disorder. Research in Autism Spectrum Disorders, 1(1): 85-100. Available at: 10.1016/j.rasd.2006.08.001.

Lewis, F.M., G.C. Woodyatt and B.E. Murdoch, 2007. Linguistic and pragmatic language skills in adults with autism spectrum disorder: A pilot study. Research in Autism Spectrum Disorders, 2(1): 176-187. Available at: 10.1016/j.rasd.2007.05.002.

Mayes, S.D., S.L. Calhoun and E.W. Crowell, 2000. Learning disabilities and ADHD: Overlapping spectrum disorders. Journal of Learning Disabilities, 33(5): 417-424. Available at: https://doi.org/10.1177/002221940003300502.

Newton, A.T., A.D.I. Kramer and D.N. McIntosh, 2009. Autism online: A comparison of word usage in bloggers with and without autism spectrum disorders. In Proceedings of the SIGCHI Conference on Human Factors in Computing Systems. ACM. pp: 463-466.

Prince-Hughes, D., 2002. Aquamarine blue 5: Personal stories of college students with autism. Athens, OH: Swallow Press.

Randolph, J.J., 2005. Free-marginal multirater Kappa: An alternative to Fleiss' fixed-marginal multirater Kappa. Paper Presented at the Joensuu University Learning and Instruction Symposium 2005, Joensuu, Finland, October 14-15th, 2005. (ERIC Document Reproduction Service No. ED490661).

Rutter, M., A. Le Couteur and C. Lord, 2005. Autism diagnostic interview-revised. Los Angeles: Western Psychological Services.

Schairer, K.S. and N.W. Nelson, 1996. Communicative possibilities of written conversations with adolescents who have autism. Child Language Teaching and Therapy, 12(2): 164-180. Available at: https://doi.org/10.1177/026565909601200206.

Semel, E., E.H. Wiig and W.A. Secord, 2003. Clinical evaluation of language fundamentals examiner's manual. 4th Edn., San Antonio, TX: Harcourt Assessment, Inc.

Siegel, S. and Castellan, 1988. Nonparametric statistics for the social sciences. 2nd Edn., New York: McGraw-Hill.

Sinclair, J., 2005. Autism network international: The development of a community and its culture. Available from http://www.autreat.com/History_of_ANI.html.

Warrens, M.J., 2010. Inequalities between multi-rater kappas. Advances in Data Analysis and Classification, 4(4): 271-286. Available at: https://doi.org/10.1007/s1 1634-010-0073-4. 